\title{
Critical Nonlinear Nonlocal Equations on a Half-Line
}

Elena I. Kaikina, Leonardo Guardado-Zavala,

Hector F. Ruiz-Paredes and Jesus A. Mendez Navarro

Abstract. We study nonlinear nonlocal equations on a half-line in the critical case

$$
\left\{\begin{array}{l}
\partial_{t} u+\beta|u|^{\alpha} u+\mathbb{K} u=0, \quad x>0, t>0, \\
u(0, x)=u_{0}(x), \quad x>0 \\
\partial_{x}^{j-1} u(0, t)=0, j=1, \ldots, M
\end{array}\right.
$$

where $\beta \in \mathbf{C}$. The linear operator $\mathbb{K}$ is a pseudodifferential operator defined by the inverse Laplace transform with dissipative symbol $K(p)=E_{\alpha} p^{\alpha}$, the number $M=\left[\frac{\alpha}{2}\right]$. The aim of this paper is to prove the global existence of solutions to the inital-boundary value problem (0.1) and to find the main term of the large time asymptotic representation of solutions in the critical case.

Mathematics Subject Classification (2000). 35S15; 35B40.

Keywords. Dissipative nonlinear evolution equations, large time asymptotics, fractional derivative.

\section{Introduction}

In this paper we study critical nonlinear nonlocal equations on a half-line

$$
\left\{\begin{array}{l}
\partial_{t} u+\beta|u|^{\alpha} u+\mathbb{K} u=0, \quad x>0, t>0, \\
u(0, x)=u_{0}(x), \quad x>0, \\
\partial_{x}^{j-1} u(0, t)=0, \quad j=1, \ldots, M,
\end{array}\right.
$$

where $\beta \in \mathbf{C}, \alpha>0$. The linear operator $\mathbb{K}$ is a pseudodifferential operator defined by the inverse Laplace transform as follows

$$
\mathbb{K} u=\frac{1}{2 \pi i} \int_{-i \infty}^{i \infty} e^{p x} K(p)\left(\hat{u}(p, t)-\sum_{j=1}^{[\alpha]} \frac{\partial_{x}^{j-1} u(0, t)}{p^{j}}\right) d p
$$


where

$$
\hat{u}(p)=\int_{0}^{+\infty} e^{-p x} u(x) d x
$$

denotes the Laplace transform of $u$. The symbol $K(p)$ is given by $K(p)=E_{\alpha} p^{\alpha}$, $E_{\alpha} \in \mathbf{C}$. We suppose that the operator $\mathbb{K}$ is dissipative, i.e. $\operatorname{Re}(K(p))>0$ for $\operatorname{Re}(p)=0$. Here and below $p^{\alpha}$ is the main branch of the complex analytic function in the half-complex plane $\operatorname{Re} p \geq 0$, so that $1^{\alpha}=1$ (we make a cut along the negative real axis $(-\infty, 0)$ ). The number of the boundary data $M=\left[\frac{\alpha}{2}\right]$ (see book $[3]),[\alpha]$ denotes the largest integer less then $\alpha$.

The theory of nonlinear pseudodifferential equations plays an important role in the contemporary mathematical physics(see book [11]). Such equations serve as a basis for mathematical models to describe the different phenomena in modern Physics, Biology, Technology and others fields of science. As examples, we mention famous equations of Ott-Sudan-Ostrovskiy and Whitham (see [12-14]), which are picked out from complicated natural mathematical models via the separation of the basic mechanism responsible for interesting nonlinear effects. Another example is the equation

$$
u_{t}+|u|^{2} u+\int_{0}^{+\infty} q(x-y) u(s, t) d s=0
$$

encountered in the theory of the Langmuir waves in plasma [15].

In this paper we study initial-boundary value problem (1.1) in the critical case. Recently much attention was drown to the study of the global existence and large time asymptotic behavior of solutions to the Cauchy problems for nonlinear nonlocal equations in the critical and sub critical cases (see $[2,4-7,9,10,16]$ and a literature cited therein). In the super critical case $\sigma>\alpha$ the global existence result for the initial-boundary value problem (1.1) was obtained in paper [1]. In the so-called sub critical case $\sigma \in(0, \alpha)$ initial-boundary value problem (1.1) was studied in the paper [8].

Here we give a general approach to study initial-boundary value problem in the critical nonconvective case. We now state our result.

We denote by $\mathbf{L}^{p}$ for $1 \leq p \leq \infty$, the usual Lebesgue space with a norm $\|\phi\|_{p}=\left(\int_{0}^{+\infty} \mid \phi(x)^{p} d x\right)^{1 / p}$ if $1 \leq p<\infty$ and $\|\phi\|_{\infty}=\operatorname{ess.sup}_{x \geq 0}|\phi(x)|$. Define by $\mathbf{W}_{p}^{0, a}=\left\{\phi \in \mathbf{L}^{p}:\langle x\rangle^{a} \phi \in \mathbf{L}^{p}\right\}$ weighted Sobolev space with a norm $\|\phi\|_{\mathbf{W}_{p}^{0, a}}=$ $\left\|\langle x\rangle^{a} \phi\right\|_{\mathbf{L}^{p}}$, where $\langle x\rangle=1+x$. By $\mathbf{C}(\mathbf{I} ; \mathbf{B})$ we denote the space of continuous functions from a time interval $\mathbf{I}$ to the Banach space $\mathbf{B}$. Different positive constants could be denoted by the same letter $C$.

We define $\theta=\int_{0}^{\infty} u_{0}(x) d x>0$. We denote

$$
\eta=\operatorname{Re} \beta \int_{0}^{+\infty}|\Lambda(s)|^{\alpha} \Lambda(s) d s>0,
$$


where

$$
\begin{aligned}
\Lambda(s) & =D \int_{-i \infty}^{i \infty} d z e^{s z} e^{-K(z)} \Gamma\left(\frac{\{\alpha\}}{\alpha},-K(z)\right), \\
\Gamma(\nu, a) & =\int_{a}^{+\infty} e^{-t} t^{\nu-1} d t, \quad \Gamma(\nu)=\int_{0}^{+\infty} e^{-t} t^{\nu-1} d t, \\
D & =\frac{1}{2 \pi^{2} i} \frac{(-1)^{\frac{\{\alpha\}}{\alpha}}}{1+A} \sin \left(\frac{\{\alpha\}}{\alpha}\right) \Gamma\left(\frac{[\alpha]}{\alpha}\right),
\end{aligned}
$$

and

$$
A=(-1)^{\{\alpha\}}\{\alpha\} \Gamma(-\{\alpha\}) \Gamma(\{\alpha\}) 2 i \sin \pi\{\alpha\} .
$$

Also we denote by

$$
G(t, s)=(t+1)^{-\frac{1}{\alpha}} \Lambda\left(s t^{-\frac{1}{\alpha}}\right)
$$

and

$$
g(t)=1+\theta^{\alpha} \eta \log (1+t) .
$$

In the present paper we prove the following result.

Theorem 1. Let $\alpha, \beta \in \mathbf{C}, \operatorname{Re} E_{\alpha} e^{i \frac{\pi \alpha}{2}}>0$. We assume that the initial data $u_{0} \in$ $\mathbf{W}_{1}^{0, a}, a \in(0,1)$ have a sufficiently small norm $\varepsilon=\left\|\langle x\rangle^{a} u_{0}\right\|_{1}$. Then there exists a unique solution $u(x, t) \in \mathbf{C}\left([0, \infty) ; \mathbf{W}_{1}^{0, a}\right) \cap \mathbf{C}\left((0, \infty) ; \mathbf{L}^{\infty}\right)$ of the initial-boundary value problem (1.1), satisfying the following time decay estimate

$$
\left\|g^{\frac{1}{\alpha}} u\right\|_{\mathbf{L}^{\infty}} \leq C \varepsilon .
$$

Moreover the asymptotic is true

$$
\left\|\left(u-\theta g^{-\frac{1}{\alpha}} e^{i \psi} G\right) g\right\|_{\mathbf{L}^{\infty}} \leq C,
$$

for $t \rightarrow \infty$, where $\psi(t)$ satisfies the asymptotic estimate

$$
\psi(t)=\widetilde{\eta} \log \log t+O(1)
$$

for $t \rightarrow \infty$, with some constant $\widetilde{\eta} \in \mathbf{R}$.

We organize our paper as follows. In Section 2 we give a general theory of the study critical initial-boundary value problem. Section 3 is devoted to the proof of preliminary estimates. In Section 4 we prove Theorem 1.

\section{The General approach}

Now we give a general approach for obtaining the large time asymptotic representation of solutions to the initial-boundary value problem

$$
\left\{\begin{array}{l}
u_{t}+\mathcal{N}(u)+\mathbb{K} u=0, \quad x \in \mathbf{R}^{+}, \quad t>0 \\
u(0, x)=u_{0}(x), \quad x \in \mathbf{R}^{+}, \\
\partial_{x}^{j-1} u(0, t)=0, \quad j=1, \ldots,\left[\frac{\alpha}{2}\right]
\end{array}\right.
$$


in the case of critical nonlinearity $\mathcal{N}(u)$ of nonconvective type. We fix a metric space $\mathbf{Z}$ of functions defined on $\mathbf{R}^{+}$and a complete metric space $\mathbf{X}$ of functions defined on $[0, \infty) \times \mathbf{R}^{+}$.

Definition 1. We call the function $G_{0} \in \mathbf{X}$ as the asymptotic kernel for the Green operator $\mathcal{G}$ in spaces $\mathbf{X}, \mathbf{Z}$ if there exists a continuous linear functional $f: \mathbf{Z} \rightarrow \mathbf{R}^{+}$ such that the estimate is true

$$
\left\|\langle t\rangle^{\gamma}\left(\mathcal{G}(t) \phi-G_{0}(t) f(\phi)\right)\right\|_{\mathbf{X}} \leq C\left\|_{\phi}\right\|_{\mathbf{Z}} .
$$

Definition 2. We call the nonlinearity $\mathcal{N}$ in equation (2.1) as critical nonconvective if the estimate is true

$$
\int_{0}^{t} \operatorname{Re} f\left(\mathcal{N}\left(\theta G_{0}(\tau)\right)\right) d \tau \geq \varkappa \theta^{\sigma+1} \log (1+t)
$$

for all $t>0, \theta>0$ with some positive constants $\varkappa$ and $\sigma$.

Now we prove global existence and obtain the large time asymptotic of small solutions to the problem (2.1) with a critical nonlinearity of nonconvective type. Define the function

$$
g(t)=1+\eta \theta^{\sigma} \log (1+t)
$$

with some positive constants $\eta, \theta$ and $\sigma$.

Theorem 2. Assume that the linear operator $\mathbb{K}$ is such that $f(\mathbb{K} u)=0$ for any $u \in \mathbf{X}$. Let the nonlinearity $\mathcal{N}(u)$ in equation (2.1) be critical nonconvective. Assume that

$$
e^{z} \mathcal{N}\left(u e^{-z}\right)=e^{-\sigma \operatorname{Re} z} \mathcal{N}(u)
$$

for any $z \in \mathbf{C}$ and $u \in \mathbf{X}$, where $\sigma>0$. Suppose that the estimates are valid

$$
\begin{aligned}
\mid \log (2+t) f( & \mathcal{N}(v(t))-\mathcal{N}(w(t))) \mid \\
& \leq C\{t\}^{-\alpha}\langle t\rangle^{-1}\|\log (2+t)(v(t)-w(t))\|_{\mathbf{X}}\left(\|v\|_{\mathbf{X}}^{\sigma}+\|w\|_{\mathbf{X}}^{\sigma}\right),
\end{aligned}
$$

for all $t>0$ and for any $v, w \in \mathbf{X}$, where $\alpha<1, \sigma>0$, and

$$
\begin{aligned}
& \| g(t) \int_{0}^{t} g^{-1}(\tau) \mid \mathcal{G}(t-\tau)(\mathcal{K}(v(\tau))-\mathcal{K}(w(\tau))) \mid d \tau \|_{\mathbf{X}} \\
& \leq C\|v-w\|_{\mathbf{X}}\left(\|v\|_{\mathbf{X}}+\|w\|_{\mathbf{X}}\right)^{\sigma}\left(1+\frac{\|v\|_{\mathbf{X}}+\|w\|_{\mathbf{X}}}{\theta}\right)
\end{aligned}
$$

for any $v, w \in \mathbf{X}$ such that $f(v)=f(w)=\theta>0$, where $\sigma>0$ and $\mathcal{K}(v)=$ $\mathcal{N}(v)-\frac{v}{\theta} f(\mathcal{N}(v))$. Let the initial data $u_{0} \in \mathbf{Z}$ have a small norm norm $\left\|u_{0}\right\|_{\mathbf{Z}} \leq \varepsilon$ and the mean value $\theta \equiv\left|f\left(u_{0}\right)\right| \geq C \varepsilon>0$ with some $C>0$. Then there exists a unique global solution $u \in \mathbf{X}$ to the initial problem (2.1) satisfying the time decay estimate

$$
\left\|g^{\frac{1}{\sigma}} u\right\|_{\mathbf{X}} \leq C \varepsilon .
$$

Moreover if we assume that

$$
1+\frac{\sigma}{\theta} \int_{0}^{t} \operatorname{Re} f\left(\mathcal{N}\left(\theta G_{0}(\tau)\right)\right) d \tau=g(t)+O(\log g(t))
$$


for $t \rightarrow \infty$, then the asymptotic is true

$$
\left\|\left(u-\theta g^{-\frac{1}{\sigma}} e^{i \psi} G_{0}\right) g\right\|_{\mathbf{X}} \leq C,
$$

where $\psi(t)$ satisfies the asymptotic estimate

$$
\psi(t)=\widetilde{\eta} \log \log t+O(1)
$$

for $t \rightarrow \infty$, with some constant $\widetilde{\eta} \in \mathbf{R}$.

Proof. We make a change of the dependent variable $u(t, x)=v(t, x) e^{-\varphi(t)+i \psi(t)}$ in equation (2.1). Then in view of condition (2.3) we get the following equation for the new unknown function $v(t, x)$

$$
v_{t}+\mathbb{K} v+e^{-\sigma \varphi} \mathcal{N}(v)-\left(\varphi^{\prime}-i \psi^{\prime}\right) v=0 .
$$

We now choose the auxiliary functions $\varphi(t)$ and $\psi(t)$ by the following condition

$$
f\left(e^{-\sigma \varphi} \mathcal{N}(v)-\left(\varphi^{\prime}-i \psi^{\prime}\right) v\right)=0,
$$

then in view of the condition $f(\mathbb{K} v)=0$ we obtain a conservation law

$$
\frac{d}{d t} f(v(t))=f\left(v_{t}(t)\right)=0
$$

hence $f(v(t))=f\left(v_{0}\right)$ for all $t>0$. We also choose $\varphi(0)=0$ and $\psi(0)=\arg f\left(u_{0}\right)$ so that

$$
f(v(t))=f\left(v_{0}\right)=\left|f\left(u_{0}\right)\right|=\theta>0
$$

and

$$
\varphi^{\prime}=\frac{1}{\theta} e^{-\sigma \varphi} \operatorname{Re} f(\mathcal{N}(v)), \quad \psi^{\prime}=-\frac{1}{\theta} e^{-\sigma \varphi} \operatorname{Im} f(\mathcal{N}(v)) .
$$

Thus we obtain the initial-boundary value problem for the new dependent variable $v(t, x)$

$$
\left\{\begin{array}{l}
v_{t}+\mathbb{K} v=-e^{-\sigma \varphi}\left(\mathcal{N}(v)-\frac{v}{\theta} f(\mathcal{N}(v))\right), \quad t>0, \quad x \in \mathbf{R}^{n}, \\
v(0, x)=v_{0}(x) \equiv u_{0}(x) e^{-i \arg f\left(u_{0}\right)}, \quad x \in \mathbf{R}^{n} .
\end{array}\right.
$$

We denote $h(t)=e^{\sigma \varphi(t)}$, then we get

$$
h^{\prime}=\frac{\sigma}{\theta} \operatorname{Re} f(\mathcal{N}(v)), \quad h(0)=1,
$$

hence integration with respect to time yields

$$
h(t)=1+\frac{\sigma}{\theta} \int_{0}^{t} \operatorname{Re} f(\mathcal{N}(v(\tau))) d \tau .
$$

Now the integral equation associated with (2.7) can be written as

$$
v(t)=\mathcal{G}(t) v_{0}-\int_{0}^{t} \mathcal{G}(t-\tau) \mathcal{K}(v(\tau)) \frac{d \tau}{h_{v}(\tau)},
$$

where the nonlinearity

$$
\mathcal{K}(v(\tau))=\mathcal{N}(v(\tau))-\frac{v(\tau)}{\theta} f(\mathcal{N}(v(\tau)))
$$


and the functional

$$
h_{v}(t)=1+\frac{\sigma}{\theta} \int_{0}^{t} \operatorname{Re} f(\mathcal{N}(v(\tau))) d \tau
$$

We now prove the existence of the solution $v(t, x)$ for integral equation $(2.8)$ by the contraction mapping principle. We define the transformation $\mathcal{M}(w)$ by the formula

$$
\mathcal{M}(w)=\mathcal{G}(t) v_{0}-\int_{0}^{t} \mathcal{G}(t-\tau) \mathcal{K}(w(\tau)) \frac{d \tau}{h_{w}(\tau)}
$$

for any $w \in \mathbf{B}$, where

$$
\begin{aligned}
\mathbf{B}=\{w \in \mathbf{X}: f(w)= & \theta,\|w\|_{\mathbf{X}} \leq C \varepsilon \\
& \left.\left\|\log (2+t)\left(w-\theta G_{0}(t)\right)\right\|_{\mathbf{X}} \leq C \varepsilon, \sup _{t>0} \frac{g(t)}{h_{w}(t)} \leq 3\right\},
\end{aligned}
$$

where $h_{w}(t)=1+\frac{\sigma}{\theta} \int_{0}^{t} \operatorname{Re} f(\mathcal{N}(w(\tau))) d \tau$. First we check that the mapping $\mathcal{M}$ transforms the set B into itself. Since

$$
f(\mathcal{K}(w))=f(\mathcal{N}(w))-\frac{1}{\theta} f(\mathcal{N}(w)) f(w)=0,
$$

we see that

$$
f(\mathcal{M}(w))=f\left(\mathcal{G}(t) v_{0}\right)=f\left(v_{0}\right)=\theta .
$$

By the definition of the asymptotic kernel we have

$$
\begin{aligned}
\left\|\log (2+t)\left(\mathcal{G}(t) v_{0}-\theta G_{0}(t)\right)\right\|_{\mathbf{X}} & \leq C\left\|\langle t\rangle^{\gamma}\left(\mathcal{G}(t) v_{0}-\theta G_{0}(t)\right)\right\|_{\mathbf{X}} \\
& \leq C\left\|v_{0}\right\|_{\mathbf{z}} \leq C \varepsilon
\end{aligned}
$$

and by the condition of the theorem we get

$$
\begin{aligned}
\| \log (2+t) & \left|\int_{0}^{t} \mathcal{G}(t-\tau) \mathbb{K} w(\tau) \frac{d \tau}{h_{w}(\tau)}\right| \|_{\mathbf{X}} \\
& \leq 3\left\|\log (2+t) \int_{0}^{t} g^{-1}(\tau)|\mathcal{G}(t-\tau) \mathcal{K}(w(\tau))| d \tau\right\|_{\mathbf{X}} \\
& \leq \frac{C}{\eta \theta^{\sigma}}\|w\|_{\mathbf{X}}^{\sigma+1}\left(1+\frac{\|w\|_{\mathbf{X}}}{\theta}\right) \leq C \varepsilon .
\end{aligned}
$$

In particular, we see that

$$
\begin{aligned}
\|\mathcal{M}(w)\|_{\mathbf{X}} & \leq\left\|\mathcal{G}(t) v_{0}\right\|_{\mathbf{X}}+\left\|\int_{0}^{t} \mathcal{G}(t-\tau) \mathcal{K}(w(\tau)) \frac{d \tau}{h_{w}(\tau)}\right\|_{\mathbf{X}} \\
& \leq C \varepsilon+C\left\|\int_{0}^{t} g^{-1}(\tau)|\mathcal{G}(t-\tau) \mathcal{K}(w(\tau))| d \tau\right\|_{\mathbf{X}} \leq C \varepsilon+C \varepsilon^{\sigma+1} \leq C \varepsilon,
\end{aligned}
$$


and

$$
\begin{aligned}
\left\|\log (2+t)\left(\mathcal{M}(w)-\theta G_{0}(t)\right)\right\|_{\mathbf{X}} \leq & \left\|\log (2+t)\left(\mathcal{G}(t) v_{0}-\theta G_{0}(t)\right)\right\|_{\mathbf{X}} \\
& +\left\|\log (2+t) \int_{0}^{t} \mathcal{G}(t-\tau) \mathcal{K}(w(\tau)) \frac{d \tau}{h_{w}(\tau)}\right\|_{\mathbf{X}} \\
\leq & C \varepsilon .
\end{aligned}
$$

It remains to prove the estimate

$$
h_{\mathcal{M}(w)}(t)=1+\frac{\sigma}{\theta} \int_{0}^{t} \operatorname{Re} f(\mathcal{N}(\mathcal{M}(w))) d \tau \geq \frac{1}{3} g(t)
$$

for all $t>0$. We have by condition $(2.2)$

$$
\begin{aligned}
1+\frac{\sigma}{\theta} \int_{0}^{t} \operatorname{Re} f(\mathcal{N}(\mathcal{M}(w))) d \tau= & 1+\frac{\sigma}{\theta} \int_{0}^{t} \operatorname{Re} f\left(\mathcal{N}\left(\theta G_{0}(\tau)\right)\right) d \tau \\
& +\frac{\sigma}{\theta} \int_{0}^{t} \operatorname{Re} f(\mathcal{N}(\mathcal{M}(w)) \\
& \left.-\mathcal{N}\left(\theta G_{0}(\tau)\right)\right) d \tau \geq \frac{1}{2} g(t)+R(t),
\end{aligned}
$$

where

$$
R(t)=\frac{\sigma}{\theta} \int_{0}^{t} \operatorname{Re} f\left(\mathcal{N}(\mathcal{M}(w))-\mathcal{N}\left(\theta G_{0}(\tau)\right)\right) d \tau .
$$

By estimate (2.4), we have

$$
\begin{aligned}
|R(t)| \leq & C\left\|\log (2+t)\left(\mathcal{M}(w)-\theta G_{0}(t)\right)\right\|_{\mathbf{X}}\left(\|\mathcal{M}(w)\|_{\mathbf{X}}^{\sigma}+\left\|\theta G_{0}\right\|_{\mathbf{X}}^{\sigma}\right) \\
& \times \int_{0}^{t}\{\tau\}^{-\alpha}\langle\tau\rangle^{-1} \frac{d \tau}{\log (2+\tau)} \leq C \varepsilon^{\sigma+1} \log \log (4+\tau) .
\end{aligned}
$$

Therefore by virtue of (2.10) we find that

$$
h_{\mathcal{M}(w)}(t) \geq \frac{1}{2} g(t)-C \varepsilon^{\sigma+1} \log \log (4+\tau) \geq \frac{1}{3} g(t)
$$

for all $t>0$. Thus we see that $\mathcal{M}$ transforms $\mathbf{B}$ into itself. Now by virtue of (2.5) let us estimate the difference

$$
\begin{aligned}
\| \mathcal{M}(v)- & \mathcal{M}(w) \|_{\mathbf{X}} \\
= & \left\|\int_{0}^{t} \mathcal{G}(t-\tau)\left(\mathcal{K}(v(\tau)) \frac{1}{h_{v}(\tau)}-\mathcal{K}(w(\tau)) \frac{1}{h_{w}(\tau)}\right) d \tau\right\|_{\mathbf{X}} \\
\leq & C\left\|\int_{0}^{t} g^{-1}(\tau)|\mathcal{G}(t-\tau)(\mathcal{K}(v(\tau))-\mathcal{K}(w(\tau)))| d \tau\right\|_{\mathbf{X}} \\
& +C\left\|\int_{0}^{t} g^{-1}(\tau)|\mathcal{G}(t-\tau) \mathcal{K}(w(\tau))| \frac{\left|h_{v}(\tau)-h_{w}(\tau)\right|}{g(\tau)} d \tau\right\|_{\mathbf{X}} \\
\leq & C\|v-w\|_{\mathbf{X}}\left(\varepsilon^{\sigma}+\frac{1}{\theta}\left\|\int_{0}^{t} g^{-1}(\tau)|\mathcal{G}(t-\tau) \mathcal{K}(w(\tau))| d \tau\right\|_{\mathbf{X}}\right) \\
\leq & C \varepsilon^{\sigma}\left(1+\frac{\varepsilon}{\theta}\right)\|v-w\|_{\mathbf{X}} \leq \frac{1}{2}\|v-w\|_{\mathbf{X}},
\end{aligned}
$$


where in view of (2.4) with $\nu(t)=1$ we used the estimate

$$
\begin{aligned}
\frac{\left|h_{v}(\tau)-h_{w}(\tau)\right|}{g(\tau)} & \leq \frac{C}{\theta g(t)}\left|\int_{0}^{t} f(\mathcal{N}(v(\tau))-\mathcal{N}(w(\tau))) d \tau\right| \\
& \leq \frac{C \varepsilon^{\sigma} \log (1+t)}{\theta g(t)}\|v-w\|_{\mathbf{X}} \leq \frac{C}{\theta}\|v-w\|_{\mathbf{X}} .
\end{aligned}
$$

Therefore $\mathcal{M}$ is a contraction mapping in the closed set $\mathbf{B}$ of a complete metric space $\mathbf{X}$. Hence there exists a unique global solution $v \in \mathbf{B}$ to the Cauchy problem (2.7) such that

$$
\|v\|_{\mathbf{X}} \leq C \varepsilon, \quad\left\|\log (2+t)\left(v-\theta G_{0}(t)\right)\right\|_{\mathbf{X}} \leq C \varepsilon, \quad h_{v}(t) \geq \frac{1}{3} g(t) .
$$

Using the relation $u(t, x)=v(t, x) e^{i \psi(t)} h_{v}^{-\frac{1}{\sigma}}(t)$ we obtain existence of the solution to the Cauchy problem (2.1), satisfying the following time decay estimates

$$
\left\|g^{\frac{1}{\sigma}} u\right\|_{\mathbf{x}} \leq C \varepsilon \text {. }
$$

We now prove the asymptotic for the solution. By condition (2.6) and representation (2.10) we have

$$
\begin{aligned}
h_{v}(t)= & 1+\frac{\sigma}{\theta} \int_{0}^{t} \operatorname{Re} f(\mathcal{N}(v)) d \tau \\
= & 1+\frac{\sigma}{\theta} \int_{0}^{t} \operatorname{Re} f\left(\mathcal{N}\left(\theta G_{0}(\tau)\right)\right) d \tau \\
& +\frac{\sigma}{\theta} \int_{0}^{t} \operatorname{Re} f\left(\mathcal{N}(v)-\mathcal{N}\left(\theta G_{0}(\tau)\right)\right) d \tau \\
= & g(t)+O(\log \log (4+t)) .
\end{aligned}
$$

Then via formulas $u(t, x)=e^{-\varphi(t)+i \psi(t)} v(t, x)=h_{v}^{-\frac{1}{\sigma}}(t) e^{i \psi(t)} v(t, x)$ we find the estimate

$$
\left\|\log (2+t)\left(u-\theta G_{0} e^{-i \psi} g^{-\frac{1}{\sigma}}\right)\right\|_{\mathbf{X}} \leq C,
$$

since

$$
\begin{aligned}
& \| \frac{g^{1+\frac{1}{\sigma}}}{\log \log (4+t)}\left(\theta G_{0} e^{-i \psi}\right. \\
& \left.\quad\left(h_{v}^{-\frac{1}{\sigma}}-g^{-\frac{1}{\sigma}}\right)\right) \|_{\mathbf{X}} \\
& \leq C \sup _{t>0}\left|\frac{g^{1+\frac{1}{\sigma}}}{\log \log (4+t)}\left(h_{v}^{-\frac{1}{\sigma}}-g^{-\frac{1}{\sigma}}\right)\right|\left\|G_{0}\right\|_{\mathbf{X}} \leq C .
\end{aligned}
$$

Also we have

$$
\begin{aligned}
\psi(t) & =\arg f\left(u_{0}\right)-\frac{1}{\theta} \int_{0}^{t} h_{v}^{-1}(\tau) \operatorname{Im} f(\mathcal{N}(v(\tau))) d \tau \\
& =\arg f\left(u_{0}\right)-\frac{1}{\theta} \int_{0}^{t} g^{-1}(\tau) \operatorname{Im} f\left(\mathcal{N}\left(\theta G_{0}(\tau)\right)\right) d \tau
\end{aligned}
$$




$$
\begin{aligned}
& +O\left(\int_{0}^{t}\{\tau\}^{-\alpha}\langle\tau\rangle^{-1} \frac{\log \log (4+\tau) d \tau}{\log ^{2}(2+\tau)}\right) \\
= & \widetilde{\eta} \log \log t+O(1)
\end{aligned}
$$

for large $t \rightarrow \infty$, with some constant $\widetilde{\eta} \in \mathbf{R}$. This completes the proof of Theorem 2 .

\section{Preliminaries}

We consider the following linear initial-boundary value problem on half-line

$$
\left\{\begin{array}{l}
u_{t}+\mathbb{K} u=f, \quad(x, t) \in \mathbf{R}^{+} \times \mathbf{R}^{+}, \\
u(x, 0)=u_{0}(x), \quad x \in \mathbf{R}^{+}, \\
\partial_{x}^{j-1} u(0, t)=0 \text { for } j=1,2, \ldots, M,
\end{array}\right.
$$

where $M=\left[\frac{\alpha}{2}\right]$. We have the following proposition (see book [3,8].)

Proposition 1. The solution $u(x, t)$ of the problem (3.1) has the following integral representation

$$
u(t)=\mathcal{G}(t) u_{0}+\int_{0}^{t} \mathcal{G}(t-\tau) f(\tau) d \tau,
$$

where the Green operator $\mathcal{G}(t)$ is given by

$$
\mathcal{G}(t) \phi=t^{-\frac{1}{\alpha}} \int_{0}^{+\infty} G\left(x t^{-\frac{1}{\alpha}}, y t^{-\frac{1}{\alpha}}\right) \phi(y) d y
$$

with a Green function $G(s, q)$

$$
\begin{aligned}
G(s, q)= & \frac{1}{2 \pi i} \int_{-\infty}^{i \infty} e^{z s-K(z)} d z \\
& -\frac{1}{4 \pi^{2}}\left(-E_{\alpha}\right)^{\frac{\{\alpha\}}{\alpha}} \int_{-i \infty}^{i \infty} d z e^{s z} z^{\{\alpha\}} \int_{-i \infty}^{i \infty} d \xi e^{\xi} \xi^{-\frac{\{\alpha\}}{\alpha}} \frac{f\left(\frac{z}{\phi_{1}(\xi)}, q\right)}{K(z)+\xi}
\end{aligned}
$$

where

$$
f\left(s_{1}, q\right)=\sum_{k=1}^{m} \frac{r_{k}^{-[\alpha]}}{P_{m}^{\prime}\left(r_{k}\right)} e^{-\phi_{k}(\xi) q} \prod_{l=1, l \neq k}^{m}\left(s_{1}-r_{l}\right), r_{k}=\exp \left\{\frac{i 2 \pi k}{\alpha}\right\}
$$

and

$$
\phi_{k}(\xi)=\left(\frac{\xi}{E_{\alpha}} \exp (i \pi(2 k-1))\right)^{\frac{1}{\alpha}}, \quad k=1 \ldots, m
$$

and

$$
P_{m}(u)=\prod_{j=1}^{m}\left(u-r_{j}\right)
$$


Denote by

$$
\begin{aligned}
\Lambda(s) & =D \int_{-i \infty}^{i \infty} d z e^{s z} e^{-K(z)} \Gamma\left(\frac{\{\alpha\}}{\alpha},-K(z)\right), \\
D & =\frac{1}{2 \pi^{2} i} \frac{(-1)^{\frac{\{\alpha\}}{\alpha}}}{1+A} \sin \left(\frac{\{\alpha\}}{\alpha}\right) \Gamma\left(\frac{[\alpha]}{\alpha}\right)
\end{aligned}
$$

and

$$
A=(-1)^{\{\alpha\}}\{\alpha\} \Gamma(-\{\alpha\}) \Gamma(\{\alpha\}) 2 i \sin \pi\{\alpha\} .
$$

We first collect some preliminary estimates of the Green operator $\mathcal{G}(t)$ in the norms $\|\phi\|_{\mathbf{L}^{p}}$ and $\|\phi\|_{\mathbf{L}^{1, a}}$, where $a \in(0,1), r=1, \infty$. We have the following Lemma (see [10]).

Lemma 1. The following estimates are true, provided that the right-hand sides are finite:

$$
\left\|\langle\cdot\rangle^{b} \mathcal{G}(t) \phi\right\|_{\mathbf{L}^{p}} \leq C\left(t^{-\frac{1}{\alpha}\left(1-\frac{1}{p}\right)}\langle t\rangle^{\frac{b}{\alpha}}\|\phi\|_{\mathbf{L}^{1}}+t^{-\frac{1}{\alpha}\left(\frac{1}{q}-\frac{1}{p}\right)}\langle t\rangle^{\frac{b}{2}}\left\|\langle\cdot\rangle^{b} \phi\right\|_{\mathbf{L}^{q}}\right)
$$

and

$$
\left\|(\cdot)^{b}\left(\mathcal{G}(t) \phi-\vartheta(t+1)^{-\frac{1}{\alpha}} G\left((\cdot)(t+1)^{-\frac{1}{\alpha}}\right)\right)\right\|_{\mathbf{L}^{p}} \leq C\langle t\rangle^{\frac{b-a}{\alpha}} t^{-\frac{1}{\alpha}\left(\frac{1}{q}-\frac{1}{p}\right)}\left\|\langle\cdot\rangle^{a} \phi\right\|_{q}
$$

for all $t>0$, where $1 \leq q \leq p \leq \infty, b \in[0, a], a \in(0,1), \vartheta=\int_{\mathbf{R}^{+}} \phi d x$.

Define the function

$$
g(t)=1+k \log (1+t), \quad k>0 .
$$

Now we prove the following results

Lemma 2. Let the function $f(t, x)$ have the zero mean value $\hat{f}(t, 0)=0$ Then the following inequality is valid

$$
\left\|g(t) \int_{0}^{t} g^{-1}(\tau) \mathcal{G}(t-\tau) f(\tau) d \tau\right\|_{\mathbf{X}} \leq C\|\langle t\rangle f\|_{\mathbf{X}}
$$

where

$$
\|\phi\|_{\mathbf{X}}=\sup _{t>0}\left(t^{\frac{1}{\alpha}}\|\phi(t)\|_{\mathbf{L}^{\infty}}+\langle t\rangle^{-\frac{a}{\alpha}}\left\|(\cdot)^{a} \phi(t)\right\|_{\mathbf{L}_{1}}+\|\phi(t)\|_{\mathbf{L}^{1}}\right), \quad a \in[0,1) .
$$

Proof. In view of the estimate $g^{-1}(\tau) \leq C$ and Lemma 1 we get

$$
\begin{aligned}
\left\|\int_{0}^{t} g^{-1}(\tau) \mathcal{G}(t-\tau) f(\tau) d \tau\right\|_{\mathbf{L}^{\infty}} & +\left\|\int_{0}^{t} g^{-1}(\tau) \mathcal{G}(t-\tau) f(\tau) d \tau\right\|_{\mathbf{L}^{1, a}} \\
& \leq C \int_{0}^{4}(t-\tau)^{-\frac{1}{\alpha}}\|f(\tau)\|_{\mathbf{L}^{1}} d \tau \\
& \leq C\|(1+t) f(t, x)\|_{\mathbf{X}} \int_{0}^{4}(t-\tau)^{-\frac{1}{\alpha}}(1+\tau)^{-1} d \tau \\
& \leq C g^{-1}(t)\|(1+t) f(t, x)\|_{\mathbf{X}}
\end{aligned}
$$


for all $0 \leq t \leq 4$. We now consider $t>4$. Via the condition of the lemma for the function $g(t)$ we have the estimate $(1+t)^{-\frac{a}{2 \rho}} \leq C g^{-1}(t)$ and

$$
\begin{aligned}
\sup _{\tau \in[\sqrt{t}, t]} g^{-1}(\tau) & \leq C(1+\kappa \log (1+\sqrt{t}))^{-1} \\
& \leq C\left(1+\frac{\kappa}{2} \log (1+t)\right)^{-1} \leq C g^{-1}(t) .
\end{aligned}
$$

Since $\hat{f}(t, 0)=0$ we have $\mathcal{G}(t) f=\mathcal{G}(t) f-\hat{f}(0) G(t)$. Applying the second estimate of Lemma 1 we obtain

$$
\begin{aligned}
\left\|\int_{0}^{t} g^{-1}(\tau) \mathcal{G}(t-\tau) f(\tau) d \tau\right\|_{\mathbf{L}^{p}} \\
\leq C \int_{0}^{\sqrt{t}}(t-\tau)^{\frac{1}{\alpha}\left(\frac{1}{p}-1\right)-\frac{a}{\alpha}} \tau^{\frac{a}{\alpha}-1} d \tau \sup _{\tau>0}(1+\tau)^{-\frac{a}{\rho}}\|(1+\tau) f(\tau)\|_{\mathbf{L}^{1, a}} \\
\quad+C g^{-1}(t) \int_{\sqrt{t}}^{\frac{t}{2}}(t-\tau)^{\frac{1}{\alpha}\left(\frac{1}{p}-1\right)-\frac{a}{\alpha}} \tau^{\frac{a}{\alpha}-1} d \tau \sup _{\tau>0}(1+\tau)^{-\frac{a}{\alpha}}\|(1+\tau) f(\tau)\|_{\mathbf{L}^{1, a}} \\
\\
+C g^{-1}(t) \int_{\frac{t}{2}}^{t} \tau^{\frac{1}{\alpha}\left(\frac{1}{p}-1\right)-1} d \tau \sup _{\tau>0}(1+\tau)^{-\frac{1}{\alpha}\left(\frac{1}{p}-1\right)}\|(1+\tau) f(\tau)\|_{\mathbf{L}^{p}} \\
\leq C\left(t^{\frac{1}{\alpha}\left(\frac{1}{p}-1\right)-\frac{a}{2 \alpha}}+g^{-1}(t) t^{\frac{1}{\alpha}\left(\frac{1}{p}-1\right)}\right)\|(1+t) f(t, x)\|_{\mathbf{X}} \\
\leq C g^{-1}(t) t^{\frac{1}{\alpha}\left(\frac{1}{p}-1\right)}\|(1+t) f(t, x)\|_{\mathbf{X}}
\end{aligned}
$$

for $1 \leq p \leq \infty$, and

$$
\begin{aligned}
\left\|\int_{0}^{t} g^{-1}(\tau) \mathcal{G}(t-\tau) f(\tau) d \tau\right\|_{\mathbf{L}^{1, a}} \\
\leq C \int_{0}^{\sqrt{t}} \tau^{\frac{a}{\alpha}-1} d \tau \sup _{\tau>0}(1+\tau)^{-\frac{a}{\alpha}}\|f(\tau)\|_{\mathbf{L}^{1, a}} \\
\quad+C g^{-1}(t) \int_{\sqrt{t}}^{t} \tau^{\frac{a}{\alpha}-1} d \tau \sup _{\tau>0}(1+\tau)^{-\frac{a}{\alpha}}\|f(\tau)\|_{\mathbf{L}^{1, a}} \\
\leq C\left(t^{\frac{a}{2 \alpha}}+g^{-1}(t) t^{\frac{a}{\alpha}}\right)\|(1+t) f(t, x)\|_{\mathbf{X}} \\
\leq C g^{-1}(t) t^{\frac{a}{\alpha}}\|(1+t) f(t, x)\|_{\mathbf{X}}
\end{aligned}
$$

for all $t>4$. Hence the result of the lemma follows. Lemma 2 is proved.

\section{Proof of Theorem 1}

The local existence of solutions for the initial-value problem (1.1) can be obtained by the standard contraction mapping principle (for the proof see [1]).

Theorem 3. Let the initial data $u_{0} \in \mathbf{W}_{1}^{0, a}$. Then for some $T>0$ there exists a unique solution $u(t, x) \in \mathbf{C}\left([0, T) ; \mathbf{W}_{1}^{0, a}\right) \cap \mathbf{C}\left((0, T) ; \mathbf{L}^{\infty}\right)$ of the initial-value 
problem (1.1). Moreover if the initial data are sufficiently small $\left\|\langle\cdot\rangle^{a} u_{0}\right\|_{1} \leq \varepsilon$, then there exists a time $T \geq 1$ such that the solution $u(t, x)$ of the problem (1.1) satisfy the estimates

$$
t^{\frac{1}{\alpha}}\|u(t)\|_{\mathbf{L}^{\infty}} \leq 2 \varepsilon
$$

By this theorem, it follows that the global solution (if it exists) is unique. Indeed, suppose that there exist two global solutions with the same initial data, which are different at some time $t>0$. By virtue of the continuity of solutions with respect to time, we can find a maximal time segment $[0, T]$, where the solutions are equal, but for $t>T$ they are different. Now we apply the local existence theorem taking the initial time $T$ and obtain that these solutions coincide on some interval $\left[T, T_{1}\right]$, which gives us a contradiction with the fact that $T$ is a maximal time until which the solutions coincide. So our main purpose in the proof of Theorem 1 is to show the global in time existence of solutions.

To apply Theorem 2 we choose the space

$$
\mathbf{Z}=\left\{\phi \in \mathbf{L}_{1}^{0, a}\left(\mathbf{R}^{+}\right)\right\}
$$

with $a \in(0,1]$ and the space

$$
\mathbf{X}=\left\{\phi \in \mathbf{C}([0, \infty) ; \mathbf{Z}) \cap \mathbf{C}\left((0, \infty) ; \mathbf{L}^{\infty}\right):\|\phi\|_{\mathbf{X}}<\infty\right\}
$$

with the norm

$$
\|\phi\|_{\mathbf{X}}=\sup _{t>0}\left(t^{\frac{1}{\alpha}}\|\phi(t)\|_{\mathbf{L}^{\infty}}+\langle t\rangle^{-\frac{a}{\alpha}}\left\|(\cdot)^{a} \phi(t)\right\|_{\mathbf{L}^{1}}+\|\phi(t)\|_{\mathbf{L}^{1}}\right) .
$$

Denote

$$
\begin{aligned}
G(s, t) & =D(t+1)^{-\frac{1}{\alpha}} \int_{-i \infty}^{i \infty} d z e^{s(t+1)^{\frac{1}{\alpha}} z} e^{-K(z)} \Gamma\left(\frac{\{\alpha\}}{\alpha},-K(z)\right), \\
D & =\frac{1}{2 \pi^{2} i} \frac{(-1)^{\frac{\{\alpha\}}{\alpha}}}{1+A} \sin \left(\frac{\{\alpha\}}{\alpha}\right) \Gamma\left(\frac{[\alpha]}{\alpha}\right)
\end{aligned}
$$

and

$$
A=(-1)^{\{\alpha\}}\{\alpha\} \Gamma(-\{\alpha\}) \Gamma(\{\alpha\}) 2 i \sin \pi\{\alpha\} .
$$

From Lemma 1 we have that the function $G(s, t)$ is the asymptotic kernel for the Green operator $\mathcal{G}$ in spaces $\mathbf{X}, \mathbf{Z}$ with a continuous linear functional $f$ :

$$
f(\phi)=\int_{\mathbf{R}^{+}} \phi(x) d x
$$

Denote by

$$
g(t)=1+\eta \theta^{\alpha} \log (1+t)
$$


where

$$
\begin{aligned}
\eta & =\operatorname{Re}|\beta| \int_{\mathbf{R}^{+}} \Lambda^{\alpha+1}\left(x(\tau+1)^{-\frac{1}{\alpha}}\right) d x>0, \\
\Lambda(s) & =D \int_{-i \infty}^{i \infty} d z e^{s z} e^{-K(z)} \Gamma\left(\frac{\{\alpha\}}{\alpha},-K(z)\right) . \\
\mathcal{N}(\phi) & =\beta|\phi|^{\alpha} \phi .
\end{aligned}
$$

By a direct computation we have

$$
\begin{aligned}
1+\frac{\alpha}{\theta} & \int_{0}^{t} \operatorname{Re} f(\mathcal{N}(\theta G(\tau))) d \tau \\
& =1+\operatorname{Re}|\beta| \theta^{\alpha} \int_{0}^{t} \int_{\mathbf{R}^{+}}(\tau+1)^{-1-\frac{1}{\alpha}} \Lambda^{\alpha+1}\left(x(\tau+1)^{-\frac{1}{\alpha}}\right) d x d \tau=g(t)
\end{aligned}
$$

and

$$
e^{z} \mathcal{N}\left(u e^{-z}\right)=e^{z}\left|u e^{-z}\right|^{\alpha} u e^{-z}=e^{-\alpha z} \mathcal{N}(u),
$$

hence conditions (2.2), (2.3) and (2.6) are fulfilled. Since by interpolation inequality

$$
\begin{aligned}
\log (2+t)\|\mathcal{N}(v(t))-\mathcal{N}(w(t))\|_{\mathbf{L}^{1}} \leq & C \log (2+t)\left(\|v(t)\|_{\mathbf{L}^{\infty}}^{\alpha}\right. \\
& \left.+\|w(t)\|_{\mathbf{L}^{\infty}}^{\alpha}\right)\|v(t)-w(t)\|_{\mathbf{L}^{1}} \\
\leq & C\langle t\rangle^{-1}\|\log (2+t)(v-w)\|_{\mathbf{X}}\left(\|v\|_{\mathbf{X}}^{\sigma}+\|w\|_{\mathbf{X}}^{\sigma}\right),
\end{aligned}
$$

hence condition (2.4) is true. From Lemma 2 we have if $\int_{\mathbf{R}^{+}} f(t, x) d x=0$ then the following inequality is valid

$$
\left\|g(t) \int_{0}^{t} g^{-1}(\tau) \mathcal{G}(t-\tau) f(\tau) d \tau\right\|_{\mathbf{X}} \leq C\|f\|_{\mathbf{Y}},
$$

where $\|f\|_{\mathbf{Y}}=\|\langle t\rangle f\|_{\mathbf{X}}$. Also we have for $\mathcal{K}(\phi)=\mathcal{N}(\phi)-\frac{\phi}{\theta} \int_{\mathbf{R}^{+}} \mathcal{N}(\phi) d x$.

$$
\begin{aligned}
\|\mathcal{K}(v)-\mathcal{K}(w)\|_{\mathbf{Y}} \leq & \|\mathcal{N}(v)-\mathcal{N}(w)\|_{\mathbf{Y}} \\
& +\frac{1}{\theta}\left(\|v\|_{\mathbf{X}}+\|w\|_{\mathbf{X}} \sup _{t>0}\{t\}^{\frac{1}{\alpha}}\langle t\rangle\|\mathcal{N}(v(t))-\mathcal{N}(w(t))\|_{\mathbf{L}^{1}}\right. \\
& +\frac{1}{\theta}\|v-w\|_{\mathbf{X}} \sup _{t>0}\{t\}^{\frac{1}{\alpha}}\langle t\rangle\left(\|\mathcal{N}(v(t))\|_{\mathbf{L}^{1}}+\|\mathcal{N}(w(t))\|_{\mathbf{L}^{1}}\right) \\
\leq & C\|v-w\|_{\mathbf{X}}\left(\|v\|_{\mathbf{X}}^{\alpha}+\|w\|_{\mathbf{X}}^{\alpha}\right)\left(1+\frac{1}{\theta}\|v\|_{\mathbf{X}}+\frac{1}{\theta}\|w\|_{\mathbf{X}}\right) .
\end{aligned}
$$

Therefore we see that condition (2.5) is fulfilled. Now applying Theorem 2 we easily get the results of Theorem 1 .

\section{Acknowledgements}

This work was supported in part by CONACYT. 


\section{References}

[1] R. E. Cardiel, E. I. Kaikina and P. I. Naumkin, Asymptotics for nonlinear nonlocal equations on a half-line, Communications in Contemporary Mathematics, 8 (2006), no. $2,189-219$.

[2] A. Gmira and L. Veron, Large time behavior of the solutions of a semilinear parabolic equation in $\mathbf{R}^{N}$, J. Diff. Eq., 53 (1984), 258-276.

[3] N. Hayashi and E. I. Kaikina, Nonlinear theory of pseudodifferential equations on a half-line, North-Holland Mathematics Studies, 194 (2004), 340.

[4] N. Hayashi, E. I. Kaikina, P. I. Naumkin, I. A. Shishmarev, Asymptotics for dissipative nonlinear equations. Lecture Notes in Mathematics, 1884. Springer Verlag, Berlin, 2006, pp. 557.

[5] N. Hayashi, E. I. Kaikina and P. I. Naumkin, Large time behavior of solutions to the dissipative nonlinear Schrödinger equation, Proceedings of the Royal Soc. Edingburgh, 130A (2000), 1029-1043.

[6] N. Hayashi, E. I. Kaikina and P. I. Naumkin, Global existence and time decay of small solutions to the Landau-Ginzburg type equations, Journal d'Analyse Mathematique, 90 (2003), 141-173.

[7] N. Hayashi, E. I. Kaikina and P. I. Naumkin, Landau-Ginzburg type equations in the sub critical case, Commun. Contemp. Math., 5 (1) (2003), 127-145.

[8] E. I. Kaikina, Subcritical nonlinear nonlocal equations on a half-line, Journal of Mathematical Analysis and Applications, 305 (2005), 316-346.

[9] S. Kamin and L. A. Peletier, Large time behaviour of solutions of the heat equation with absorption, Ann. Scuola Norm. Sup. Pisa, 12 (1985), 393-408.

[10] O. Kavian, Remarks on the large time behavior of a nonlinear diffusion equation, Ann. Inst. Henri Poincaré, Analyse non linéaire, 4 (5) (1987), 423-452.

[11] P. I. Naumkin, I. A. Shishmarëv, Nonlinear nonlocal equations in the theory of waves, Translations of Mathematical Monographs, 133. American Mathematical Society, Providence, RI, 1994.

[12] L. A. Ostrovsky, Short-wave asymptotics for weak-shock waves and solitons in Mechanics, Int. J. Non-Linear Mechanics, 11 (1976), 401-416.

[13] E. Ott and R. N. Sudan, Nonlinear theory of ion acoustic waves with Landau damping, Phys. Fluids, 12, no. 11 (1969), 2388-2394.

[14] G. B. Whitham, Linear and Nonlinear Waves, Wiley, New York, 1974.

[15] V.E. Zakharov, Collapse of the Langmuir Waves, Soviet Phys. JETP, 35 (1972), $1745-1759$

[16] E. Zuazua, Some recent results on the large time behavior for scalar parabolic conservation laws, in Elliptic and parabolic poblems. Proc. 2nd European Conference. Pitman Res. Notes Math. Ser., 325 (1995), 251-263. 
Elena I. Kaikina

Instituto de Matemáticas

UNAM Campus Morelia, AP 61-3 (Xangari)

Morelia CP 58089, Michoacán

Mexico

e-mail: ekaikina@matmor.unam.mx

Leonardo Guardado-Zavala, Hector F. Ruiz-Paredes and Jesus A. Mendez Navarro Posgrado en Electrica

Instituto Tecnológico de Morelia

CP 58120, Morelia, Michoacán

Mexico

e-mail: guardado@ps.itm.mx

hruiz@sirio.tsemor.mx

Received: 8 September 2008.

Accepted: 19 November 2008. 